\title{
Cost savings from a telemedicine model of care in northern Queensland, Australia
}

\section{Darshit A Thake MB BS, FRACP. ClinDipPallMed Medical Oncologist \\ Richard Monypenny BIngAgr, PhD, Adjunct Associate Professor ${ }^{2}$ \\ Ian Olver \\ $\mathrm{MD}, \mathrm{PhD}$ \\ Chief Executive Officer \\ Sabe Sabesan BM BS, FRACP Director, ' and Clinical Dean \\ 1Department of Medical Oncology, Townsville Hospital \\ Townsville, QLD. \\ 2 School of Business. \\ James Cook University, Townsville, QLD. \\ 3 Cancer Council of Australia \\ Sydney, NSW. \\ 4 Townsville Clinical School, \\ School of Medicine and Dentistry, \\ James Cook University. \\ Townsville, QLD. \\ sabe_sabesan@ \\ health.qld.gov.au}

MJA 2013; 199: 414-417 doi: 10.5694/mjal2.1178

Online first 9/09/13

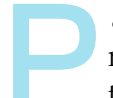

atients with cancer who live in rural and remote areas of Australia and other countries with large rural populations travel long distances to major centres to receive specialist care. ${ }^{1,2}$ Some of these patients require overnight accommodation for themselves and their escorts. In Australia, all or part of the travel costs and part of the accommodation costs are usually borne by jurisdictional governments. ${ }^{3}$ Telemedicine has the potential to provide specialist consultations to patients in their home towns and minimise the need for distant travel, although evidence for the relative costs and benefits of telemedicine is mixed. ${ }^{4-7}$

Townsville Cancer Centre (TCC) provides tertiary cancer care to people living in northern Queensland, Australia. As people living in this area must travel long distances to receive cancer care, the Medical Oncology department at TCC embarked on a teleoncology model of care for its rural satellite sites in 2007. ${ }^{8}$ Questionnairebased satisfaction surveys carried out in 2009 found this model was acceptable to patients and health professionals. $^{9}$

The Townsville teleoncology model involves videoconference sessions in which medical oncologists consult with patients who may be supported during the videoconferences by local health care professionals. Referrals to the teleoncology service are managed by a coordinator at TCC. The need for local health care professionals to be present during videoconferences is determined by the complexity of the cases. This means that, in some cases, patients attend videoconferences alone. Consultations may involve review of new cases, follow-up of existing cases or urgent review for acutely ill patients, usually within 24 hours of request. New cases usually take up to 30-40 minutes and reviews take about 10-20 minutes.

We aimed to conduct a cost-savings analysis of the teleoncology model of care at the TCC compared with the usual care model. This analysis was

\begin{abstract}
Objective: To conduct a cost analysis of a telemedicine model for cancer care (teleoncology) in northern Queensland, Australia, compared with the usual model of care from the perspective of the Townsville and other participating hospital and health services.
\end{abstract}

Design: Retrospective cost-savings analysis; and a one-way sensitivity analysis performed to test the robustness of findings in net savings.

Participants and setting: Records of all patients managed by means of teleoncology at the Townsville Cancer Centre (TCC) and its six rural satellite centres in northern Queensland, Australia between 1 March 2007 and 30 November 2011.

Main outcome measures: Costs for set-up and staffing to manage the service, and savings from avoidance of travel expenses for specialist oncologists, patients and their escorts, and for aeromedical retrievals.

Results: There were 605 teleoncology consultations with 147 patients over 56 months, at a total cost of $\$ 442276$. The cost for project establishment was $\$ 36000$, equipment/maintenance was $\$ 143271$, and staff was $\$ 261520$. The estimated travel expense avoided was $\$ 762394$; this figure included the costs of travel for patients and escorts of $\$ 658760$, aeromedical retrievals of $\$ 52400$ and travel for specialists of $\$ 47634$, as well as an estimate of accommodation costs for a proportion of patients of $\$ 3600$. This resulted in a net saving of $\$ 320$ 118. Costs would have to increase by $72 \%$ to negate the savings.

Conclusion: The teleoncology model of care at the TCC resulted in net savings, mainly due to avoidance of travel costs. Such savings could be redirected to enhancing rural resources and service capabilities. This teleoncology model is applicable to geographically distant areas requiring lengthy travel.

performed from the perspective of savings to the Townsville and other participating hospital and health services.

\section{Methods}

Demographic details of patients managed by means of teleoncology between 1 March 2007 and 30 November 2011 were collected from the oncology database of the TCC. Data collected included age, sex, ethnicity, type of consultation and diagnosis.

\section{Costs}

We considered project establishment and equipment costs to be "one-off" costs, and maintenance, communication and staff costs to be "running" costs. ${ }^{10,11}$ Cost calculations for the study period are summarised in Box 1 . One-off costs: The total equipment cost was \$23 726 per centre (Queensland Health unpublished data), which included a camera (\$16700), an LCD monitor (\$1200), a cart (\$1020), wall mount brackets (\$300), a shelf for holding the camera (\$156) and miscellaneous costs such as consumables (\$100), freight (\$1000), one-off license cost (\$250), installation (\$2350), and assembly, testing and implementation (\$600).

Installation costs varied depending on the location and complexity of the project; they included the travel costs of a telecommunications service provider of $\$ 3000$, and installation of power and data cabling ranging from $\$ 1000$ to $\$ 4000$, depending on the area. ${ }^{11}$ The average satellite installation cost was $\$ 6000$ per centre.

Running costs: Maintenance costs included the salary and costs of travel to and accommodation at each site for technical experts performing system monitoring and annual check-ups. As technical experts are employed regardless of telehealth, their salaries added no extra cost to our model. The average annual cost for travel to and accommodation at each site for one technical expert was $\$ 750$. 
1 Costs of the Townsville teleoncology model over 56 months from 1 March 2007 to 30 November 2011

\begin{tabular}{lccc} 
Type of cost & Cost per centre & Cost for six centres & Total \\
\hline Project establishment & $\$ 6000$ & $\$ 6000 \times 6$ & $\$ 36000$ \\
Equipment & $\$ 20376$ & $\$ 20376 \times 6$ & $\$ 122256$ \\
Maintenance & $\$ 750$ per year & $\$ 750 \times 6 \times 4.6$ & $\$ 21015$ \\
Communication & 0 & 0 & 0 \\
Teleoncology coordinator for TCC & $\$ 48000$ per year & $\$ 48000 \times 4.6$ & $\$ 224160$ \\
Nurse in Mt Isa (O.1 FTE) & $\$ 8000$ per year & $\$ 8000 \times 4.6$ & $\$ 37360$ \\
Total cost for the study period & & & $\$ 442276$ \\
\hline TCC $=$ Townsville Cancer Centre. FTE = full-time equivalent. & &
\end{tabular}

The cost of establishing telehealth networks also generated no additional costs, as video connectivity within the Queensland Health network uses the same link as all other data sources.

Staffing costs included the annual salary and overheads of employing the teleoncology coordinator for 3 days a week - \$48000 per year. The role of the coordinator was to receive referrals from doctors and coordinate appointments at rural and tertiary venues. Additionally, as the population of Mt Isa (a mining town approximately $900 \mathrm{~km}$ inland from Townsville) is sufficiently large to support having a chemotherapy nurse sit in with patients during sessions one morning a week, there was a cost of $\$ 8000$ per year for the nurse's salary.

\section{Savings}

Savings in our model were attributed to: - avoiding travel by patients and escorts to a tertiary centre;

- avoiding overnight accommodation for patients and escorts in Townsville;

- avoiding aeromedical retrievals; and - avoiding travel by specialist oncologists.

Savings from avoiding travel by patients to a tertiary centre were calculated by multiplying return travel cost for two people (the patient and one escort) by the number of consultations at every satellite site; as determined and fully reimbursed by the Queensland Health Patient Travel Subsidy Scheme. ${ }^{3}$ Proserpine was not included in calculations as travel to and from there involved a 3-hour road trip in a privately owned car and did not require overnight accommodation.

Under the usual care model, overnight accommodation in Townsville after treatment was required, on average, by $10 \%$ of patients. Normally,
Queensland Health reimburses 30\% of the accommodation cost with the patient paying the remainder. Hence, a cost for overnight accommodation was calculated as required for $10 \%$ of the total number of consultations.

Seeing patients urgently by means of videoconferencing and advising the necessary management plan to local medical services avoided aeromedical retrieval of patients from satellite sites to the tertiary centre, thus representing further savings.

Finally, regular 3-weekly visits to satellite sites by a specialist oncologist became unnecessary. We based savings calculations for specialist travel and accommodation on the same prices used to calculate costs for patient travel and accommodation.

\section{Exclusions}

Costs excluded from the calculations of costs and savings were:

- the social cost of disruption to patient work routine, family routine and loss of income;

- indirect benefits, such as prevention of loss of wages by patients and relatives and reduction in workload at the home site;

- loss of time incurred by specialists during travel to the satellites (on average, a specialist would spend 6 hours for a return trip between Townsville and Mt Isa, including time spent at the airport and on the plane);

- the cost of staff (other than the new teleoncology coordinator and a nurse) at the tertiary centre and in the six satellite sites, who were employed regardless of the teleoncology model.

One-way sensitivity analysis was performed to test the robustness of the findings in net savings. ${ }^{10}$ This analysis was based on a number of assumptions about contributing variables. The robustness and extent to which

\section{Statistical analysis}

the findings could be generalised were explored by varying the values given to the variables in the one-way sensitivity analysis.

\section{Ethics approval}

The project was approved by Townsville Hospital Ethics Committee (HREC/12/QTHS/29).

\section{Results}

There were 605 consultations with 147 patients between TCC and six satellite centres from 1 March 2007 to 30 November 2011. The remoteness of the centres, and distribution of patients and consultations per centre are shown in Box 2. Ninety-two per cent of all consultations were with patients in Mt Isa and Proserpine. Patients were about equally distributed by sex, with 69 men $(47 \%)$ and 78 women $(53 \%)$, and $24(16 \%)$ were Indigenous. A wide variety of cancer types were seen, with breast cancer being the most common (31\%), followed by lung cancer (18\%), gastrointestinal cancers $(8 \%)$, genitourinary cancers $(7 \%)$, melanoma $(8 \%)$ and other cancers $(16 \%)$. There were 54 consultations in the first year of the project (2007-2008). This number increased to 129 in 2008-2009, 136 in 2009-2010, and 286 in 2010-2011. The number of new patients enrolled increased each year (25 in 2007-2008; 31 in 2008-2009; 37 in 2009-2010; and 54 in 2010-2011).

Over the period of the study, four patients from Mt Isa required urgent consultations, which were performed either on the day of or within 24 hours of referral. Before the teleoncology clinics began, these patients would have required transfer to TCC. There have been no interhospital transfers from Mt Isa since the teleoncology clinics began. Details of savings realised are shown in Box 3.

Our analysis showed that total cost of the teleoncology project in the first year was $\$ 115825$, while savings were $\$ 59195$. In the second year, there were only running costs of $\$ 45457$ while, as a result of an increased number of consultations, the savings were $\$ 157929$. In the third year (2009-2010), four new centres were started, and the total cost of the establishment and running of the centres 


$\begin{aligned} & 2 \text { Remoteness of the centres and distribution of patients and consultations per } \\
& \text { centre }\end{aligned}$
\begin{tabular}{lccc} 
Teleoncology centres & $\begin{array}{c}\text { Distance from } \\
\text { Townsville }\end{array}$ & $\begin{array}{c}\text { No. of } \\
\text { patients }\end{array}$ & $\begin{array}{c}\text { No. of } \\
\text { consultations }\end{array}$ \\
\hline Mt Isa & $900 \mathrm{~km}$ & $122(82 \%)$ & $516(85 \%)$ \\
Proserpine & $200 \mathrm{~km}$ & $14(10 \%)$ & $40(7 \%)$ \\
Hughenden & $400 \mathrm{~km}$ & 2 & 11 \\
Winton & $600 \mathrm{~km}$ & 4 & 21 \\
Doomadgee & $1200 \mathrm{~km}$ & 1 & 3 \\
Gulf of Carpentaria & $1400 \mathrm{~km}$ & 4 & 14 \\
(Normanton, Mornington Island, Karumba) & & 147 & 605 \\
Total & & &
\end{tabular}

was \$221302 against savings of $\$ 164795$. In 2010-2011, the cost of running the existing centres was $\$ 59692$, while savings amounted to $\$ 380475$ as a result of a significant increase in the number of consultations. Overall, the total cost of the teleoncology project over 56 months was \$442276, while the estimated expense avoided was $\$ 762394$; this represents a net saving of $\$ 320118$.

The break-even point (the point at which costs equal savings) varied depending on distance, patient numbers and the complexity of cases managed. For example, in Mt Isa, the establishment and running costs in the first 12 months were $\$ 75926$. At a travel cost of $\$ 1000$ for a patient and escort, excluding overnight accommodation, savings were generated after 76 consultations. This means that smaller towns and towns closer to the major centres with low patient numbers will take longer to generate savings, or may not generate savings at all. Overall, under the
TCC teleoncology model, initial costs were negated after 105 consultations at Mt Isa.

Net savings: The net saving over 56 months was $\$ 320118$. Therefore, the costs would have to increase from $\$ 442276$ to $\$ 762394$ (ie, by $72 \%$ ) for the net savings to decrease to zero.

As the net savings were large, making the small increase in cost variables unlikely to affect the outcome, we did not proceed with a sophisticated sensitivity analysis.

Equipment use: While the equipment purchased and installed was for teleoncology services, it is now used by other services for more than $50 \%$ of the time. As we attributed the establishment and equipment cost entirely to the teleoncology service in our analysis, the cost was an overestimation.

Travel with escort: We assumed that all patients travelled with escorts. However, taking the example of $\mathrm{Mt}$

\section{Sensitivity analysis}

\begin{tabular}{|c|c|c|}
\hline Description of expenses prevented & Calculation of cost & Total \\
\hline Return travel cost for patient and one escort to Townsville* & & $\$ 658760$ \\
\hline Mt Isa & $516 \times 2 \times \$ 600=\$ 619200$ & \\
\hline Hughenden & $11 \times 2 \times \$ 260=\$ 5720$ & \\
\hline Winton & $21 \times 2 \times \$ 320=\$ 13440$ & \\
\hline Doomadgee & $3 \times 2 \times \$ 1150=\$ 6900$ & \\
\hline Normanton & $8 \times 2 \times \$ 480=\$ 7680$ & \\
\hline Mornington Island & $4 \times 2 \times \$ 580=\$ 4640$ & \\
\hline Palm Island & $1 \times 2 \times \$ 110=\$ 220$ & \\
\hline Karumba & $1 \times 2 \times \$ 480=\$ 960$ & \\
\hline Overnight accommodation in Townsville ${ }^{\dagger}$ ( $10 \%$ of total consultations) & $\$ 60 \times 2 \times 30$ & $\$ 3600$ \\
\hline Urgent aeromedical retrieval of four patients from Mt Isa & $\$ 13100 \times 4$ & $\$ 52400$ \\
\hline Specialist/registrar travel once every 3 weeks for 56 months $^{\ddagger}$ & $17 \times \$ 600 \times 4.67$ & $\$ 47634$ \\
\hline Total savings for the study period & & $\$ 762394$ \\
\hline
\end{tabular}

* Number of consultations $\times 2$ (patient and escort) $\times$ return travel cost. $\dagger 10 \%$ of total consultations $\times 2$ (patient and escort) $\times$ the subsidy amount. $\ddagger$ Number of visits per year $\times$ return travel cost.
Isa (the largest centre), if we assume that only half of the 516 patients from there travelled with escorts, the cost of travel decreases by $\$ 154800$, leaving a net saving of $\$ 165318$.

Air travel cost: We used the lowest price available in our calculation, but a proportion of the specialist oncologist and patient travel is booked only a few days before travel, costing two to three times the lowest price. Therefore, our analysis probably underestimated this cost.

\section{Discussion}

The TCC model of cancer care is one example of the use of telemedicine to facilitate the provision of specialist cancer services to rural patients. It reduces travel for patients and doctors, reduces interhospital transfers and provides access to ongoing medical education for staff working in remote areas by improving access to specialist oncologists. ${ }^{12}$ However there are drawbacks such as possible depersonalisation, excessive dependence on technology and increased clinical risk (eg, supervision and management of side effects of chemotherapy remotely), although early safety analysis results from our model show promising results. ${ }^{13,14}$

Evidence for cost-effectiveness of telemedicine services in comparison with conventional face-to-face consultations is mixed. A 2012 systematic review concluded that there was no conclusive evidence that telemedicine and telecare interventions were costeffective compared with conventional health care over 20 years. ${ }^{4}$ However, the studies in this systematic review varied in their methods of cost analysis, patient travel distances, number of patients served, types of specialties involved and extent of services provided, making it difficult to arrive at firm conclusions.

Studies in Kansas in the United States reported that the telemedicine cost for cancer care was lower than the face-to-face clinic cost and that the cost of telemedicine clinics had declined over the years due to increase in patient numbers. ${ }^{6,15}$ Similarly, a study from Queensland, Australia, reported savings from paediatric telemedicine clinics. ${ }^{7}$ In 
contrast, a US cost analysis reported no cost benefit for a telemedicine model that provided various specialty services to eight rural centres in Arizona; this was attributed to low patient numbers. ${ }^{5}$

Like the previous studies from Kansas $^{6}$ and Queensland, ${ }^{7}$ our study shows significant savings to the health system. We found that small changes in cost were unlikely to affect the outcome because there were large net savings. Therefore, a simple oneway sensitivity analysis was adequate for the purpose our study. The major contributor to cost savings was avoiding travel by patients and their escorts and specialist oncologists. In comparison with other studies, the models in the earlier Kansas ${ }^{6}$ and Queensland ${ }^{7}$ studies and in our study served patients from very long distances and in larger numbers.

In our model, in Mt Isa, all the medical oncology services were able to be provided locally by telehealth, which avoided interhospital transfers and led to further cost savings. However, our findings may not be generalisable to models with smaller patient numbers and with patients travelling smaller distances. Since July 2011, more than $80 \%$ of our consultations have been eligible for a Medicare rebate by the Australian government. While we did not include this in our cost analysis, these rebates would provide further financial benefit to the hospital and health services from the telehealth model.
At TCC, the number of consultations doubled every year. Mt Isa and Proserpine had large increases in the number of consultations as these centres also provided increasingly complex chemotherapy treatments over time. Other centres, where such large growth did not occur, may not generate savings because of smaller patient numbers. For these centres, the decision to continue the service should be based on equity of access and social justice, not on economic grounds. At smaller rural centres, sharing of the system by more than one specialty will be likely to improve the savings further.

In conclusion, the Townsville teleoncology model saves money for participating health service districts while providing cancer care to rural northern Queensland closer to patients' homes. The main driver of net savings is avoidance of travel costs for patients and their escorts and for specialists. Ideally, net savings should be redirected to further improving rural infrastructure and capabilities.

Acknowledgements: We acknowledge the help of Barbara Van Houts, Statewide Telehealth Services, Queensland Health and Renelle Malone, Patient Travel Office, Townsville Hospital, for help with collecting cost details.

Competing interests: No relevant disclosures.

Provenance: Not commissioned; externally peer reviewed.

Received 6 Dec 2012, accepted 27 Mar 2013.

1 Underhill C, Bartel R, Goldstein D, et al. Mapping oncology services in regional and rural Australia. Aust J Rural Health 2009; 17: 321-329.

2 Doolittle GC, Spaulding AO. Providing access to oncology care for rural patients via telemedicine. J Oncol Pract 2006; 2: 228-230.
3 Queensland Health. Patient Travel Subsidy Scheme. http://www.health.qld.gov.au/ptss (accessed Feb 2013).

4 Mistry H. Systematic review of studies of the cost-effectiveness of telemedicine and telecare. Changes in the economic evidence over twenty years. J Telemed Telecare 2012; 18: 1-6.

5 de la Torre A, Hernández-Rodriguez C, García L. Cost analysis in telemedicine: empirical evidence from sites in Arizona. J Rural Health 2004; 20: 253-257.

6 Doolittle GC, Williams AR, Spaulding A, et al. A cost analysis of a tele-oncology practice in the United States. J Telemed Telecare 2004; 10 Suppl 1: 27-29.

7 Smith AC, Scuffham P, Wootton R. The costs and potential savings of a novel telepaediatric service in Queensland. BMC Health Serv Res 2007; 7: 35.

8 Sabesan S, Brennan S. Teleoncology for cancer care in rural Australia. Chapter 13. In: Graschew G, Rakowsky S, editors. Telemedicine techniques and applications [internet]. InTech, 2011: 289306. http://www.intechopen.com/books/ telemedicine-techniques-and-applications/ tele-oncology-for-cancer-care-in-rural-australia (accessed Apr 2013).

9 Sabesan S, Simcox K, Marr I. Medical oncology clinics through videoconferencing: an acceptable telehealth model for rural patients and health workers. Intern Med J 2012; 42: 780-785.

10 Drummond MF, Sculpher MJ, Torrance GW, et al. Methods for the economic evaluation of health care programmes. 3rd ed. Oxford: Oxford University Press, 2005.

11 Crowe BL. Cost-effectiveness analysis of telemedicine. J Telemed Telecare 1998; 4 Suppl 1: 14-17.

12 Sabesan S, Larkins S, Evans R, et al. Telemedicine for rural cancer care in North Queensland: bringing cancer care home. Aust J Rural Health 2012; 20: 259-264.

13 Wootton R. Telemedicine: a cautious welcome. BMJ 1996; 313: 1375-1377.

14 Chan B, Sabesan S. Safety of tele-oncology and chemotherapy delivery in rural centres [abstract]. Proceedings of the Clinical Oncology Society of Australia Annual Scientific meeting; 2012 Nov 13-15; Brisbane, Australia. http://cosaipos-2012.m.asnevents.com.au/event/abstract/ 2677\%20last\%20accessed\%2022/7/2013 (accessed Feb 2013).

15 Doolittle GC, Harmon A, Williams A, et al. A cost analysis of a tele-oncology practice. J Telemed Telecare 1997; 3 Suppl 1: 20-22. 